\title{
Blended Interaction Design: A Spatial Workspace Supporting HCI and Design Practice*
}

\author{
Florian Geyer \\ Human-Computer Interaction Group, University of Konstanz \\ Universitätsstraße 10, 78464 Konstanz, Germany \\ florian.geyer@uni-konstanz.de
}

\begin{abstract}
This research ${ }^{1}$ investigates novel methods and techniques along with tool support that result from a conceptual blend of human-computer interaction with design practice. Using blending theory with material anchors as a theoretical framework, we frame both input spaces and explore emerging structures within technical, cognitive, and social aspects. Based on our results, we will describe a framework of the emerging structures and will design and evaluate tool support within a spatial, studio-like workspace to support collaborative creativity in interaction design.
\end{abstract}

Keywords: interaction design, design research, conceptual blending, blending theory, design tools, creativity support.

\section{Motivation}

Interaction design is broadly perceived as a recent trend in human-computer interaction (HCI) that emphasizes on designing user experiences with technology [1,2]. Its interpretations differ between the two converging perspectives: "Interaction design as a design discipline" and "Interaction design as an extension of HCI" [3]. In recent years, HCI research developed methods, techniques, and tools that aim on supporting interaction designers in practice. However, due to the complexity of the developed methods and inappropriate integration, application in practice remains sparse [4]. One of the reasons for this is that interaction design research "has not been grounded in or guided by a sufficient understanding and acceptance of the nature of design practice" [5, p.56]. Consequently, there is a growing interest in the role and nature of design in HCI research [6,7]. These contributions have already increased awareness in interaction design practice $[1,8]$ and will influence research over time. However, due to the tension between interaction design and HCI [3] as well as HCI and software engineering (SE) [9], attempts specifically addressed at supporting design practice in $\mathrm{HCI}$ have not yet been successful [5]. One possible solution to this discussion is the proposal to merge, or rather blend, these disciplines into a new one [10, p.198]. Tensions

\footnotetext{
* Advisor: Harald Reiterer.

${ }^{1}$ This dissertation work is supervised by Prof. Dr. Harald Reiterer, Human-Computer Interaction Group, University of Konstanz, Germany, harald.reiterer@uni-konstanz.de
} 
between design thinking and engineering philosophies may then result in a distinctive, creative domain that has emergent structures of its own.

\section{Research Approach}

In order to effectively blend design practice and HCI it is necessary to understand and describe design rationality [5], a notion that has been labeled with concepts such as the reflective practitioner [11] or the thoughtful designer [7]. More detailed descriptions of disciplined behavior of designers can be found in [1,12] and [13]. These authors show that following activities, among others, are fundamental for design rationality: Sketching is at the core of design and is used to explore relationsships between design ideas, form and function as well as big picture and details. Exploring many different alternatives in a conversational way while constantly shifting focus between ill-defined problems and solutions is the prevalent work style. Spatial structures, like design studios that make judgements visible and application of design critique techniques are the foundation of design argumentation. Eventually, process awareness is essential to deal with the complexity of design tasks.

However, HCI research struggles with integrating these designerly ways [12] into interaction design practice. One reason for this is that, due to a focus on engineering, most methods actually employed in practice impose a linear progression through design tasks [14]. Another reason is the fact that user interface design tools merely focus on the task of constructing a single built instead of supporting the messy nature of designer's explorative and spatial thinking modes [5]. A possible solution therefore has to consist of two blends, namely a blend of methods and techniques, as well as a blend of work styles and tool support.

\subsection{Theoretical Foundations}

We ground our research within blending theory, which is a recent framework developed by Fauconnier and Turner [15] to deal with online meaning construction. Except from Imaz and Benyon [10] and Markussen and Krogh [2], blending theory has received only little consideration in HCI research. To account for a deep understanding of how people construct meaning as they think and act, Fauconnier and Turner introduced the notion of "conceptual blending" [15]. They describe the basic principles as follows: "A conceptual blend operates in two input mental spaces to yield a third space, the blend. Partial structure from the input spaces is projected into the blended space, which has emergent structure of its own." [15, p.149]. Notable here is that the blend develops a new structure that did not exist in either of the two input spaces. This is realized by modifying existing structures through the cognitive operations of composition, completion, and elaboration. Hutchins [16] added the notion of "material anchors" to the original framework. In doing so, he claims awareness to the relevance of physical form within input spaces and the bodily interaction with the physical world. By employing both concepts as a framework for describing and mapping key features of the input spaces, we are confident to create a distinctive blend that has its own structure. Nevertheless, we also seek to provide a reference in application of blending theory as a foundation to interaction design. 


\subsection{Method and Proposed Solution}

Based on the described foundations, we are blending the two mental and physical input spaces HCI and design practice into a novel emerging structure that we call "Blended Interaction Design". In previous research, we gained valuable expertise with the integration of agile SE methods into HCI that we will bring into this input space [17]. We expect that the tension between the proposed input spaces leads to a novel, more creative perspective.

As a first step, we explored the structure of our input spaces and examined related work. We found that an important prerequisite for a successful blend is the reduction of complexity that results from the evolution of both spaces. Therefore, we decided to frame our input spaces to include only those opposing structures that we found most significant within the technical, cognitive, and social aspects.

Simulation. Visualizing design ideas and concepts is the integral activity within both input spaces that drives the design progress. While HCI has a focus on modeling and prototyping, sketching as the central design activity is more opportunistic [1]. Overall, these different activities lead to two separate perspectives on process awareness, shifting between problem space and solution as well as the generation of alternatives.

Creativity. Creative work styles in design differ fundamentally from work styles with engineering tools commonly used in HCI [14]. Most design tools do not explicitely support divergent thinking, generation of alternatives, and decision-making, which often leads to fixation effects [14] and undesired results. In contrast, design techniques emphasize on the spatial nature of synthesis in design, where juxtaposed artifacts allow reflection-in-action [11].

Collaboration. Both input spaces are interdisciplinary and inherently social domains. Therefore, a blend has to accommodate common understanding in representations, expressions, and evaluation methods. Engineering methods in HCI often bury design artifacts and decisions in file systems. Studio settings and spatial workspaces may provide a more practical environment for collaborative design.

Each of these frames has structures that contribute to the blend. We are currently applying the principles of blending theory to construct an image of a proposed solution. Based on the results, we will then develop and describe a framework of methods that emerges from the mental structures of the blend. Concurrently, we will design and implement visualization and interaction techniques that integrate the physical structures within a spatial workspace based on a computation-augmented design studio setting with interactive whiteboards, large high-resolution displays, and tabletops. Therefore, we seek to combine physical interaction concepts that built upon the principles of natural interaction (e.g. digital pen and paper, tangible design artifacts) with our object-oriented multiscale interface paradigm ZOIL [18]. Eventually, we will assess our efforts with evaluation measures and case studies. For evaluation of developed methods, we will draw upon feedback from the research community and our industrial partners while we seek to evaluate the physical workspace within lecture projects and class assignments. 


\section{Expected Contributions}

This research will contribute to interaction design and HCI research within several areas. On the one hand, we will provide reference and case studies for application of blending theory as a foundation to interaction design. On the other hand, we will contribute to the appreciation and support of design practice in HCI. Eventually, we will - based on elaborated needs for simulation, creativity support and collaboration design, and evaluate novel visualization and interaction techniques for tool support within computation-augmented design studio settings.

\section{References}

1. Buxton, B.: Sketching User Experiences: Getting the Design Right and the Right Design. Morgan Kaufmann, San Francisco (2007)

2. Markussen, T., Krogh, P.G.: Mapping Cultural Frame Shifting in Interaction Design with Blending Theory. International Journal of Design 2(2), 5-17 (2008)

3. Löwgren, J.: Interaction Design. from Interaction-Design.org (2008), http://www.interaction-design.org/encyclopedia/ interaction_design.html

4. Rogers, Y.: New Theoretical Approaches for HCI. Annual Review of Information, Science and Technology 38, 1-43 (2004)

5. Stolterman, E.: The Nature of Design Practice and Implications for Interaction Design Research. International Journal of Design 2(1), 55-65 (2008)

6. Winograd, T., Bennett, J., De Young, L., Hartfield, B.: Bringing Design to Software. Addison Wesley Professional, Reading (1996)

7. Löwgren, J., Stolterman, E.: Thoughtful Interaction Design. MIT Press, Cambridge (2004)

8. Moggridge, B.: Designing Interactions. MIT Press, Cambridge (2006)

9. Seffah, A., Gulliksen, J., Desmarais, M.C.: Human-Centered Software Engineering - Integrating Usability in the Software Development Lifecycle. Springer, Heidelberg (2005)

10. Imaz, M., Benyon, D.: Designing with Blends: Conceptual Foundations of Human Computer Interaction and Software Engineering. MIT Press, Cambridge (2007)

11. Schön, D.A.: The Reflective Practitioner: How Professionals Think in Action. Basic Books (1983)

12. Cross, N.: Designerly Ways of Knowing. Board of International Research in Design. Birkhäuser, Basel (2007)

13. Lawson, B.: How Designers Think, The Design Process Demystified. Architectural Press (2005)

14. Terry, M., Mynatt, E.D.: Recognizing Creative Needs in User Interface Design. In: Proceedings of the 4th Conference on Creativity \& Cognition, pp. 38-44 (2002)

15. Fauconnier, G., Turner, M.: The Way We Think: Conceptual Blending and the Mind's Hidden Complexities. Basic Books (2003)

16. Hutchins, E.: Material Anchors for Conceptual Blends. Journal of Pragmatics 37(10), 1555-1577 (2005)

17. Memmel, T., Geyer, F., Rinn, J., Reiterer, H.: Tool-Support for Interdisciplinary and Collaborative User Interface Specification. In: Proceedings of the IADIS IHCI (2008)

18. Jetter, H., König, W.A., Gerken, J., Reiterer, H.: ZOIL - A Cross-Platform User Interface Paradigm for Personal Information Management. In: CHI 2008 Workshop - The Disappearing Desktop: Personal Information Management (2008) 